\title{
COMPLICAÇÕES DAS OPERAÇÕES DE RECONSTRUÇÃO DO TRÂNSITO INTESTINAL
}

\author{
SILVANA MARQUES E SILVA ${ }^{1}$, CECÍLIA CARDINALE LIMA DE MELO ${ }^{1}$, SORAIA BARROSO DE ALMEIDA", \\ HERBETH FRANCO QUEIROZ ${ }^{1}$, ALOISIO FERNANDO SOARES ${ }^{2}$
}

${ }^{1}$ Residentes da Unidade de Cirurgia Geral do Hospital Regional da Asa Norte, ${ }^{2}$ Preceptor da Residência Médica de Cirurgia Geral do Hospital Regional da Asa Norte

MARQUES E SILVA S, MELO CCL, ALMEIDA SB, QUEIROZ HF, SOARES AF. Complicações das Operações de Reconstrução do Trânsito Intestinal. Rev bras Coloproct, 2006;26(1):24-27.

RESUMO: Introdução: A reconstrução do trânsito intestinal acarreta elevados índices de morbimortalidade, dependente do procedimento realizado na operação inicial, da técnica operatória necessária na reconstituição do trânsito intestinal e dos fatores de risco inerentes ao paciente. Objetivos: Determinar as características demográficas dos pacientes submetidos à reconstrução de trânsito intestinal, analisar as operações realizadas e as complicações delas decorrentes. Métodos: Análise retrospectiva dos prontuários dos pacientes submetidos à reconstrução de trânsito intestinal no Hospital Regional da Asa Norte em um período de 4 anos (20012004). Resultados: Foram incluídos 70 pacientes, sendo $34 \%$ mulheres e $66 \%$ homens, com idade média de 42,5 anos. A cirurgia tipo Hartmann foi o motivo para a reconstrução do trânsito intestinal em 45,7\% dos pacientes. A anastomose término-terminal foi realizada em 70\% dos casos. Intercorrências clínicas ocorreram em $54 \%$ dos pacientes e incluíram íleo paralítico (11,4\%), vômitos

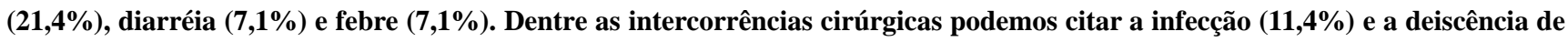
ferida operatória $(5,7 \%)$, evisceração $(2,8 \%)$, formação de fístula $(2,8 \%)$ e o abscesso intra-cavitário $(1,4 \%)$. Não houve óbitos. Conclusão: A operação para reconstrução do trânsito intestinal não é desprovida de complicações. Desta forma, é necessário uma indicação precisa para a realização da colostomia.

Descritores: cólon, colostomia, reconstrução, complicações pós-operatórias, morbidade

\section{INTRODUÇÃO}

A colostomia é realizada há quase trezentos anos no tratamento de lesões do cólon e reto. Desde esta época, sua utilização foi difundida no tratamento de outras patologias cirúrgicas, como as obstruções intestinais por neoplasia, as complicações da doença diverticular e a Síndrome de Fournier. Este procedimento é, muitas vezes, realizado por cirurgiões gerais durante intervenções cirúrgicas de urgência.

Diferente da facilidade técnica da realização da colostomia em comparação com o seu fechamento e do relativo baixo risco da sua confecção, a reconstrução do trânsito intestinal acarreta elevados índices de morbimortalidade, com taxas que variam de $0 \%$ a $4,5 \%$ e $0 \%$ a $50 \%$ respectivamente ${ }^{1,2}$. Bocic et al. ${ }^{3}$ (1999) em uma série de 132 pacientes, relataram um índice de morbidade de $36,2 \%$ e de mortalidade de $1,7 \%$. Bannura et al. ${ }^{4}$ (1999), em um estudo envolvendo 100 pacientes, demonstraram taxas de $34 \%$ e $1,7 \%$ respectivamente. Habr-Gama et al. ${ }^{5}$ (1997), avaliando 73 pacientes, demonstraram taxas de morbidade de $34,2 \%$ e de mortalidade de 3,6\%. Carreiro et al. ${ }^{6}(2000)$, analisando 35 pacientes, demonstraram morbidade de

Trabalho realizado na Unidade de Cirurgia Geral do Hospital Regional da Asa Norte (HRAN), Brasília, DF, Brasil

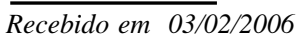

Aceito para publicação em 23/02/2006 
25,7\% e ausência de óbitos. Rey et al. ${ }^{7}$ (2000), em uma amostra de 56 pacientes, encontraram índice de morbidade de $25 \%$. Moreira et al. ${ }^{8}$ (2002), estudando 67 pacientes, obtiveram morbidade de 19,2\% .Também não houve óbitos nestes dois últimos estudos.

Estes percentuais descritos demonstram a importância da determinação de fatores que influenciam a evolução clínica dos pacientes submetidos à cirurgia de reconstrução do trânsito intestinal. Dentre estes, destaca-se o procedimento realizado na operação inicial e as complicações decorrentes deste ${ }^{7}$, a técnica operatória empregada no restabelecimento da continuidade intestinal e a experiência do cirurgião ${ }^{9,10}$, o uso consciente de antibióticos e os cuidados pré e pós operatórios ${ }^{8}$, além dos fatores de risco associados ao próprio paciente.

O objetivo deste estudo foi determinar as características demográficas dos pacientes submetidos à reconstrução de trânsito intestinal em uma instituição do Sistema Único de Saúde, além de analisar informações a respeito do procedimento cirúrgico e as complicações decorrentes.

\section{MÉTODO}

Foi realizada uma análise retrospectiva dos prontuários dos pacientes submetidos à reconstrução de trânsito intestinal no Hospital Regional da Asa Norte (HRAN) em um período de 4 anos (2001-2004). As informações colhidas eram registradas em um protocolo pré-estabelecido, incluindo dados demográficos, realização de preparo intestinal, indicação e tipo da ostomia realizada no primeiro procedimento cirúrgico, tipo de reconstrução intestinal empregada, duração do ato operatório, uso de antibióticos, drenagem da cavidade, tempo de internação, co-morbidades e complicações pósoperatórias imediatas.

As cirurgias de reconstrução de trânsito foram realizadas por 13 diferentes cirurgiões do serviço.

\section{RESULTADOS}

Foram incluídos 70 pacientes, sendo 46 do sexo masculino $(65,7 \%)$ e 24 do sexo feminino $(34,3 \%)$. A média de idade foi de 42,5 anos (16 a 92 anos).
A indicação mais comum da ostomia foi a lesão por projétil de arma de fogo $(22,9 \%)$, seguida pelo megacólon chagásico $(21,4 \%)$, neoplasia de cólon $(11,4 \%)$ e Síndrome de Fournier $(8,6 \%)$. Indicações menos freqüentes foram lesão do cólon por acidente automobilístico, neoplasia de ovário, fístula digestiva, ulcera péptica perfurada, perfuração intestinal por corpo estranho, hérnia encarcerada, etc (Tabela-1).

A maioria dos pacientes não apresentava comorbidades $(72,9 \%)$. Dentre as patologias associadas encontradas, destaca-se hipertensão arterial sistêmica (20\%), diabete mellitus $(5,71 \%)$, insuficiência renal crônica $(2,8 \%)$ e coronariopatia $(1,4 \%)$.

Os tipos de ostomia mais prevalentes foram a colostomia à Hartmann $(45,7 \%)$ e a colostomia em alça $(41,4 \%)$. A fístula colocutânea havia sido realizada em $8,6 \%$ dos casos e a ileostomia em alça em $4,3 \%$.

O preparo do cólon foi realizado em 68 pacientes $(97,1 \%)$. A técnica empregada em nosso serviço é a ingestão de manitol a 50\%, adicionado ao suco de laranja em quantidades iguais, além de "fleet" enemas ou soluções glicerinadas via retal.

As operações de reconstrução do trânsito realizadas foram anastomose boca a boca $(60 \%)$, sutura simples da parede anterior $(30 \%)$ e anastomose ileo-retal $(10 \%)$. O tempo médio de cirurgia foi de 147,6 minutos (30 a 435 minutos).

Foi realizada antibioticoprofilaxia em $45,7 \%$, sendo esta iniciada no intra-operatório em 44,3\% dos pacientes. Em 55,7\% dos casos optou-se por antibioticoterapia, sendo $30 \%$ destes de curta duração. Somente 3 pacientes $(4,3 \%)$ não fizeram uso de

Tabela 1 - Indicações da realização da colostomia.

\begin{tabular}{lrr}
\hline Indicação & N & $\%$ \\
\hline Lesão por projétil de fogo & 16 & 22,9 \\
Megacólon chagásico & 15 & 21,4 \\
Neoplasia de colon & 8 & 11,4 \\
Síndrome de Fournier & 6 & 8,6 \\
Lesão por arma branca & 4 & 5,7 \\
Diverticulite & 3 & 4,3 \\
Pós apendicectomia & 3 & 4,3 \\
Doença inflamatória intestinal & 2 & 2,8 \\
Desconhecida & 2 & 2,8 \\
Outras & 15 & 21,4 \\
\hline
\end{tabular}


Tabela 2 - Esquemas de antibiótico utilizados.

\begin{tabular}{lrr}
\hline Antibióticos & N & \% \\
\hline Cefazolina + metronidazol & 31 & 44,3 \\
Ceftriaxona + metronidazol & 9 & 12,9 \\
Gentamicina + metronidazol & 8 & 11,4 \\
Cefazolina + clindamicina & 3 & 4,3 \\
Cefazolina & 2 & 2,8 \\
Cefazolina + cloranfenicol & 2 & 2,8 \\
Outros & 12 & 7,1 \\
\hline
\end{tabular}

antibióticos durante a internação. Os esquemas de antibióticos utilizados estão listados na Tabela-2.

Não foi realizada drenagem da cavidade em 55 pacientes $(78,6 \%)$. O tempo médio de internação foi de 12,6 dias ( 5 a 42 dias).

As intercorrências clínicas ocorreram em $58,4 \%$ dos pacientes, sendo a mais comum a presença de vômitos no pós-operatório $(21,4 \%)$ (Tabela-3). Intercorrências cirúrgicas estiveram presentes em $35,5 \%$ dos pacientes.(Tabela-4)

\section{DISCUSSÃO}

A nossa amostra foi composta predominantemente por homens jovens, em idade produtiva. Este pode ser um dos fatores que influenciaram na baixa prevalência de co-morbidades encontrada. As causas mais comuns para a realização da colostomia foram perfuração por arma de fogo, o que retrata o nível de violência em nosso meio, e megacólon chagásico, uma vez que o nosso serviço é de referência para pacientes

Tabela 3 - Intercorrências clínicas pós-operatórias.

\begin{tabular}{lrc}
\hline Intercorrência & N & \% \\
\hline Vômitos & 15 & 21,40 \\
Ìleo paralítico & 8 & 11,40 \\
Diarréia & 5 & 7,10 \\
Retenção urinária & 4 & 5,70 \\
Fezes sanguinolentas & 4 & 5,70 \\
Pneumonia & 2 & 2,80 \\
Outros & 3 & 4,30 \\
Nenhuma & 29 & 41,60 \\
\hline
\end{tabular}

oriundos de regiões onde a Doença de Chagas é considerada endêmica, como cidades do Estado de Goiás e da Bahia. Outros estudos realizados demonstram resultados diferentes de acordo com sua demanda de pacientes. ${ }^{11,12,13,14,15}$

A operação para reconstrução do trânsito intestinal mais utilizada nesta série foi a anastomose boca a boca, com predomínio da anastomose colo-retal após operação de Hartmann (45,7\%).

O preparo de cólon foi realizado na maior parte dos casos. Este é um procedimento rotineiro no preparo pré-operatório dos pacientes de nosso serviço. Como descrito anteriormente, este preparo é geralmente realizado com o uso, via oral, de manitol associado ao suco de laranja. Os pacientes são internados em média um a dois dias antes da data marcada para o procedimento cirúrgico. Este fato deve ser considerado na análise do tempo médio de internação hospitalar.

A drenagem da cavidade abdominal não foi realizada na maioria das intervenções cirúrgicas, seguindo uma tendência demonstrada pela medicina baseada em evidências.

A variedade de esquemas antibióticos realizada pode ser explicada pelo número de cirurgiões envolvidos no estudo, já que, em nosso serviço, no período em que foram realizadas as cirurgias, não havia padronização para o uso de antibióticos. Desta forma, a escolha do esquema seguiu a experiência e a rotina de cada profissional envolvido.

O índice de complicações clínicas foi de 58,40\% e o de complicações cirúrgicas, de 35,5\%. Estes valores podem ser considerados elevados quando comparados a dados da literatura. É importante frisar, entretanto, que neste estudo foram incluídas intercorrências de menor

Tabela 4-Intercorrências cirúrgicas pós-operatórias.

\begin{tabular}{lrr}
\hline Intercorrência & N & \% \\
\hline Infecção de FO & 8 & 11,40 \\
Febre & 5 & 7,10 \\
Deiscência de FO & 4 & 5,70 \\
Evisceração & 2 & 2,80 \\
Fístula digestiva & 2 & 2,80 \\
Outros & 4 & 5,70 \\
Nenhuma & 45 & 64,50 \\
\hline
\end{tabular}


gravidade que, em muitos casos, não são descritas. Além disso, a colostomia à Hartmann normalmente é acompanhada de um maior número de complicações pós reconstrução do trânsito intestinal, e quase metade dos pacientes envolvidos nesta série de casos apresentavam este tipo de colostomia.

\section{CONCLUSÕES}

As operações para reconstituição do trânsito intestinal não são desprovidas de complicações, sendo necessário restringir as indicações das colostomias, principalmente as do tipo Hartmann.

SUMMARY:Colostomy closure is associated with a high morbidity and mortality rates. The risk factors are: the technical conditions at the first procedure, the utilized technique to close the colostomy and the patient status. Our goal is to retrospectively analyze the demographics of the patients who underwent colostomy closure at our institution in a four year period, (2001-2004). Seventy patients were included ( $66 \%$ males, $34 \%$ females), with a median age of 42,5 years old. A Hartmann colostomy was closed in 32 patients $(45,7 \%)$ and an end-to-end anastomosis was performed in 49 patients $(70 \%)$. Complications occurred in 38 patients (54\%) and included ileus $(11,4 \%)$, PONV $(21 \%)$, diarrhea $(7,1 \%)$ and fever $(7,1 \%)$. The surgical complications were wound infection $(11,4 \%)$, aponeurosis dehiscense $(5,7 \%)$, small bowel evisceration $(2,8 \%)$, anastomotic leak $(2,8 \%)$ and intracavitary abscess $(\mathbf{1 , 4 \%})$. There were no deaths. Conclusion: Colostomy closure is associated with high morbidity rate. It is important a very careful indication for colostomy at the first operation.

Key words: colon, colostomy, reconstruction, postoperative complications, morbidity

\section{REFERÊNCIAS BIBLIOGRÁFICAS}

1. Pittman DM, Smith LE. Complications of colostomy closure. Dis Colon Rectum 1985; 28: 836-43

2. Knox AJ, Birkett FDH, Collins CD. Closure of colostomy. Br J Surg 1971;58:559-72

3. Bocic GA, Jensen CB, Abedrapo MM, Garrido RC, Pérez GO, Cúeneo AZ. Colostomías e Ileostomías: 8 Años de experiencia clínica. Rev Hosp Clin Univ Chile 1999;10(3):195-200

4. Bannura GC, Perales CG, Contreras JP, Valencia CE, Melo CL. Reconstituición del tránsito intestinal luego de la operación de Hartmann: análisis de 100 pacientes. Rev Chil Cir1999;51(4):359-66

5. Habr-Gama A, Teixeira MG, Vieira MJF, Miléu LF, Laurino Neto R, Pinotti HW. Operação de Hartmann e suas consequiências. Rev Bras Colo-proctologia 1997; 17(1):5-10.

6. Carreiro PRL, Lázaro da Silva A, Abrantes WL. Fechamento precoce das colostomias em pacientes com trauma do reto: Um estudo prospectivo e casualizado. Rev. Coleg. Bras. Cir 2000;27 (5):298-304

7. Biondo-Simöes MLP, Brenner S, Lemos R, Duck D, Rey SD. Análise das complicaçöes pós-operatórias em decolostomias . Acta Cir. Bras 2000;vol 15(supl.3):53-7

8. Curi A, Mascarenhas JCS, Moreira Junior H, Almeida AC, Moreira JPT, Moreira $\mathrm{H}$ et al. Morbimortalidade associada à reconstruçäo do trânsito intestinal: análise de 67 casos. Rev. bras. Coloproctol 2002;22(2):88-97
9. Barnett JE, Pheils M. Closure of colostomy. Aust. N Z J Surg 1976;46: 131-3

10. Demetriades D, Pezikis A, Melissas J et al. Factors influencing the morbidity of colostomy closure. Am J Surg 1988;155:5946

11. Freitas Neto EA, Saraiva CFS. Fechamento de colostomia: análise dos resultados. Rev. Bras. Coloproctol 1981;1(2):328

12. Gomis FD, Lozano AG, Pozo D. Laparoscopic reconstruction of intestinal continuity following Hartmann's procedure. Rev Esp Enferm Dig 1998; 90(7): 499-502

13. Mosdell DM, Doberneck RC. Morbidity and mortality of ostomy closure. Am J Surg 1991;162:633-637

14. Wong RW, Rappaport WD, Witzke DB, Putnam CW, Hunter GC. Factors influencing the safety of colostomy closure in the elderly. J Surg Res 1994; 57:289-292.

15. Khoury DA, Beck DE, Opelka FG, Hicks TC, Timmcke AE, Gathright Jr JB. Colostomy closure. Dis Colon Rectum 1996; 36(6):605-609.

Endereço para correspondência:

SILVANA MARQUES E SILVA

SQS 405 Bloco B Apt. 205

70.239-020 - Brasília (DF)

Fax: (61) 3244-4271 